\title{
Applications of Forward Modeling to Refinement of Grain Orientations
}

\author{
Saransh Singh ${ }^{1}$, Asher Leff ${ }^{2}$, Mitra Taheri $^{2}$ and Marc De Graef ${ }^{1}$ \\ ${ }^{1}$ Dept. of Materials Science and Engineering, Carnegie Mellon Univ., Pittsburgh PA 15213, USA \\ ${ }^{2}$ Dept. of Materials Science and Engineering, Drexel Univ., Philadelphia, PA 19104
}

Determination of the crystal orientation from a diffraction pattern falls in the general class of inverse problems, in which one uses the results of actual observations to infer parameter values that best characterize the system under investigation, in this case the crystal orientation. Inverse problems are inherently ill-posed and often difficult to solve. The inverse of the inverse problem is the forward problem which describes the explicit relationship between given model parameters and the resulting measurements. While the inverse problem may or may not use prior information of the physical processes involved in the experiment, the forward model is a physics-based model, compatible with known laws and principles of physics. Contrary to the inverse problem, the forward problem will always have a unique solution. The accuracy and precision of the prediction will depend on the validity of the model, but a unique solution is always guaranteed. The solutions of inverse problems can be greatly improved if we have accurate forward models for these processes.

Electron backscatter diffraction (EBSD) is a popular technique for orientation mapping in crystalline materials [1]. The inverse problem of orientation determination from EBSD patterns (EBSPs) is solved using Bragg's law as the forward model, also referred to as the kinematical approximation. This produces the correct pattern geometry but the intensity distributions are not correctly reproduced. Commercial indexing packages detect Kikuchi bands using the Hough transform and index the orientation by means of lookup tables. However, electron diffraction is a dynamical process, requiring solution of a quantum mechanical problem. Recently, an alternative robust indexing approach was developed, based on full dynamical pattern simulations [2-3]; this technique is known as dictionary indexing (DI).

In this contribution, we will present a forward model for the refinement of orientations obtained from either the Hough approach or the new DI approach. The model relies on searching the local orienta-tion neighborhood and looking for a better pattern match, using either the dot product or the mutual information-based Jaccard distance as a similarity metric. Naturally, this approach requires all of the experimental EBSPs to be saved during acquisition. The local neighborhood search is performed ei-ther by a multi-resolution grid approach to uniformly sample orientation space or by using a Derivative Free Optimization (DFO) algorithm with the orientation as the optimization parameter. For the present study, we have used the Bound Optimization by Quadratic Approximation (BOBYQA) algorithm.

To test the accuracy of this method, 1, 000 patterns with known orientations were simulated and subsequently indexed using the DI method; the orientations were further refined using the two different approaches. The misorientation distribution between the true and indexed orientations as well as the refined results are presented in Fig. 1(a). The distribution clearly shows that the refinement improves the orientation, with the DFO based method performing slightly better than the multi-resolution search with 3 iterations. This is also reflected in the confidence index (CI), which is the dot product of the solution with the experimental pattern, of the matches shown in Fig. 1(b). In terms of computational efficiency, the DFO method outperforms the multi-resolution search by an order of magnitude. The algorithm was also applied to refine experimental results for a partially recrystallized Brass sample. The TSL OIM results and the BOBYQA-refined orientations are shown in Fig. 2(a)-(b), respectively. 
Apart from being able to index fine structures, the refined IPF in (b) shows noticeable color differences with respect to the IPF in (a). These orientation differences are important for subsequent computation of orientation averaged bulk material properties, geometrically necessary dislocation density, etc. [4].

References:

[1] S I Wright and B L Adams, Metallurgical Transactions A 23(3) (1992), p. 759.

[2] Y H Chen, et al, Microscopy and Microanalysis, 21(3) (2015), p. 739.

[3] S I Wright, et al, Ultramicroscopy, 159(1) (2015), p. 81.

[4] Research supported by ONR \# N00014-16-1-2821. The authors acknowledge use of the Materials Characterization Facility at Carnegie Mellon University supported by grant MCF-677785.

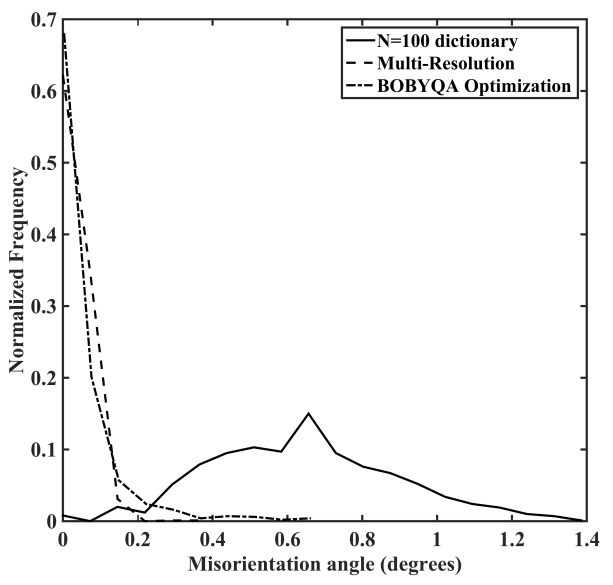

(a)

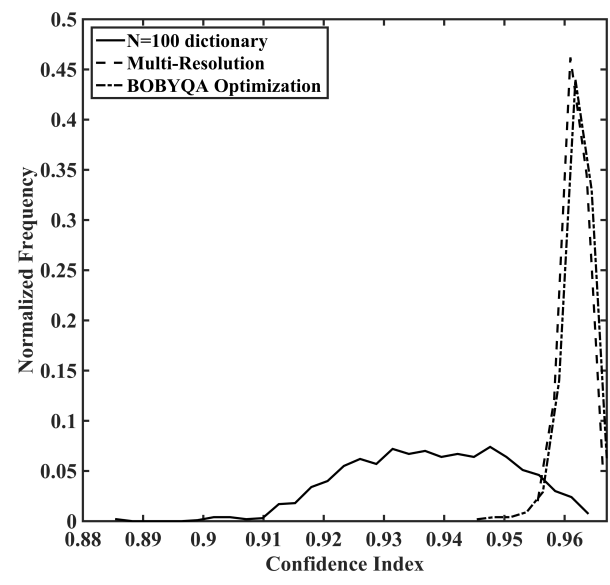

(b)

Figure 1. (a) Misorientation distribution with respect to the true orientations and (b) confidence index for $\mathrm{N}=100$ dictionary and the refined orientations using multi-resolution search and BOBYQA algorithm.

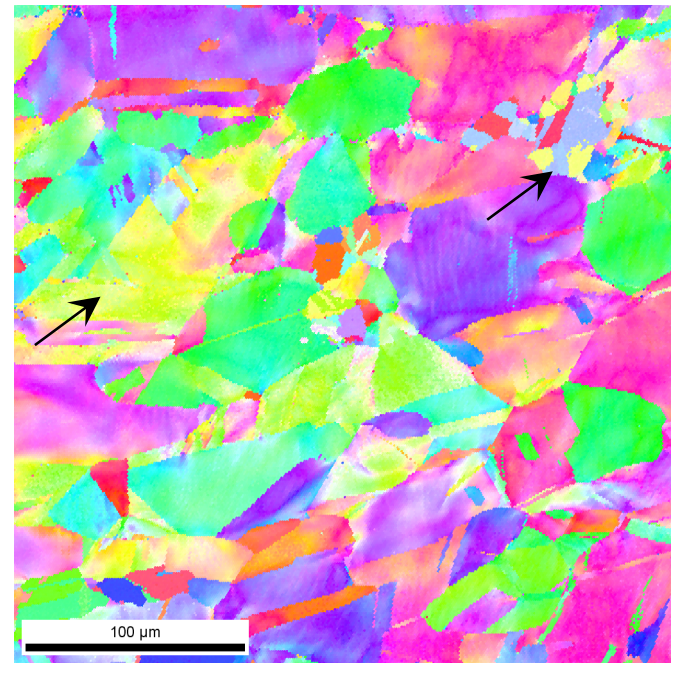

(a)

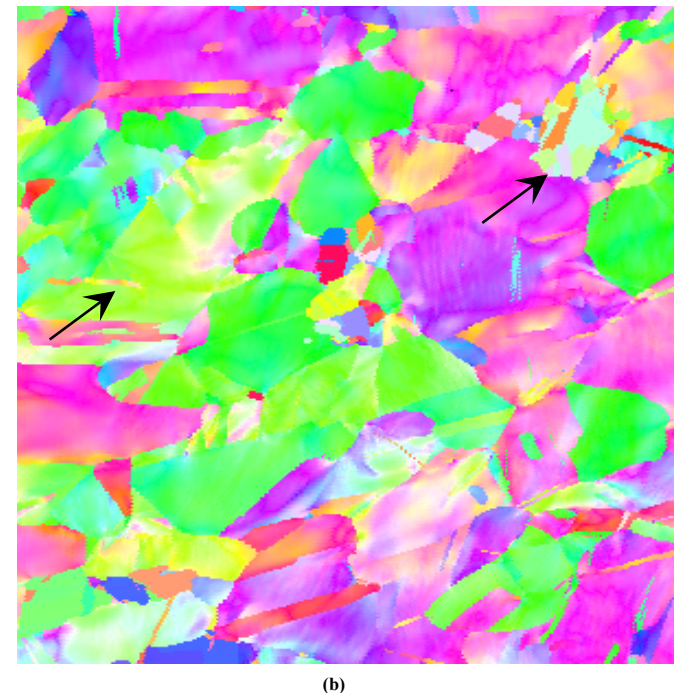

(b)

Figure 2. (001) IPF for partially recrystallized Brass using (a) TSL OIM 7 and (b) Orientation refinement using the BOBYQA algorithm. 